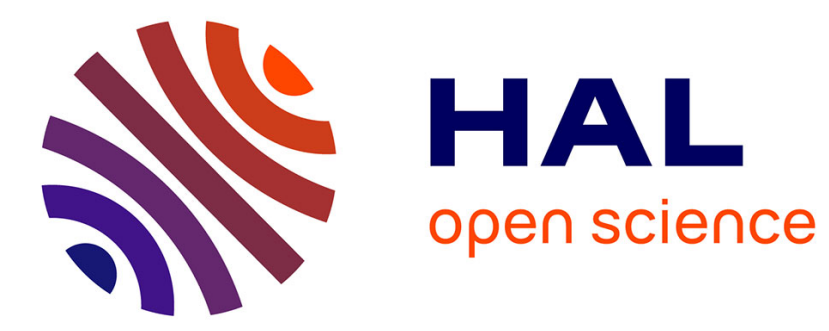

\title{
Classical elliptic current algebras. II
}

Stanislav Pakuliak, Vladimir Roubtsov, Alexey Silantyev

\section{To cite this version:}

Stanislav Pakuliak, Vladimir Roubtsov, Alexey Silantyev. Classical elliptic current algebras. II. Journal of Generalized Lie Theory and Applications, 2008, 2 (2), pp.79 - 93. 10.4172/1736-4337.1000117. hal-03054029

\section{HAL Id: hal-03054029 \\ https://univ-angers.hal.science/hal-03054029}

Submitted on 11 Dec 2020

HAL is a multi-disciplinary open access archive for the deposit and dissemination of scientific research documents, whether they are published or not. The documents may come from teaching and research institutions in France or abroad, or from public or private research centers.
L'archive ouverte pluridisciplinaire HAL, est destinée au dépôt et à la diffusion de documents scientifiques de niveau recherche, publiés ou non, émanant des établissements d'enseignement et de recherche français ou étrangers, des laboratoires publics ou privés. 


\title{
Classical elliptic current algebras. II
}

\author{
Stanislav PAKULIAK ${ }^{a}$, Vladimir RUBTSOV ${ }^{b}$ and Alexey SILANTYEV ${ }^{c}$ \\ ${ }^{a}$ Institute of Theoretical and Experimental Physics, 117259 Moscow, Russia \\ Laboratory of Theoretical Physics, JINR, 141980 Dubna, Moscow region, Russia \\ E-mail: pakuliak@theor.jinr.ru \\ ${ }^{b}$ Institute of Theoretical and Experimental Physics, 117259 Moscow, Russia \\ Départment de Mathématiques, Université d'Angers, 2 Bd. Lavoisier, 49045 Angers, France \\ E-mail: Volodya.Roubtsov@univ-angers.fr \\ ${ }^{c}$ Laboratory of Theoretical Physics, JINR, 141980 Dubna, Moscow region, Russia \\ Départment de Mathématiques, Université d'Angers, 2 Bd. Lavoisier, 49045 Angers, France \\ E-mail: silant@tonton.univ-angers.fr
}

\begin{abstract}
This is a continuation of the previous paper "Classical elliptic current algebras. I" [J. Gen. Lie Theory Appl. 2 (2002), 65-78]. We describe different degenerations of the classical elliptic algebras. They yield different versions of rational and trigonometric current algebras. We also review the averaging method of Faddeev-Reshetikhin, which allows to restore elliptic algebras from the trigonometric ones.
\end{abstract}

In memory of Leonid Vaksman

\section{Introduction}

We continue the investigation of the classical current algebras (algebras which can be described by a collection of currents) related to the classical $r$-matrices and which are quasi-classical limits of SOS-type quantized elliptic current algebras. The latter were introduced by Felder $[5,6]$. We describe shortly the results of the previous paper [14].

Let $\mathfrak{K}$ be a function algebra on a one-dimensional complex manifold $\Sigma$ with a point-wise multiplication and a continuous invariant (non-degenerate) scalar product $\langle\cdot, \cdot\rangle: \mathfrak{K} \times \mathfrak{K} \rightarrow \mathbb{C}$. We shall call the pair $(\mathfrak{K},\langle\cdot, \cdot\rangle)$ a test function algebra. The non-degeneracy of the scalar product implies that the algebra $\mathfrak{K}$ can be extended to a space $\mathfrak{K}^{\prime}$ of linear continuous functionals on $\mathfrak{K}$. We use the notation $\langle a(u), s(u)\rangle$ or $\langle a(u), s(u)\rangle_{u}$ for the action of the distribution $a(u) \in \mathfrak{K}^{\prime}$ on a test function $s(u) \in \mathfrak{K}$. Let $\left\{\epsilon^{i}(u)\right\}$ and $\left\{\epsilon_{i}(u)\right\}$ be dual bases of $\mathfrak{K}$. A typical example of the element from $\mathfrak{K}^{\prime}$ is the series $\delta(u, z)=\sum_{i} \epsilon^{i}(u) \epsilon_{i}(z)$. This is a delta-function distribution on $\mathfrak{K}$ because it satisfies $\langle\delta(u, z), s(u)\rangle_{u}=s(z)$ for any test function $s(u) \in \mathfrak{K}$.

Consider an infinite-dimensional complex Lie algebra $\mathfrak{g}$ and an operator $\hat{x}: \mathfrak{K} \rightarrow \mathfrak{g}$. The expression $x(u)=\sum_{i} \epsilon^{i}(u) \hat{x}\left[\epsilon_{i}\right]$ does not depend on a choice of dual bases in $\mathfrak{K}$ and is called a current corresponding to the operator $\hat{x}\left(\hat{x}\left[\epsilon_{i}\right]\right.$ means an action of $\hat{x}$ on $\left.\epsilon_{i}\right)$. We should interpret the current $x(u)$ as a $\mathfrak{g}$-valued distribution such that $\langle x(u), s(u)\rangle=\hat{x}[s]$. That is the current $x(u)$ can be regarded as a kernel of the operator $\hat{x}$ and the latter formula gives its invariant definition.

To describe different bialgebra structures in the current algebras we have to decompose the currents in these algebras into difference of the currents which have good analytical properties in certain domains: $x(u)=x^{+}(u)-x^{-}(u)$. The $\mathfrak{g}$-valued distributions $x^{+}(u), x^{-}(u)$ are called half-currents. To perform such a decomposition we will use so-called Green distributions [3]. Let $\Omega^{+}, \Omega^{-} \subset \Sigma \times \Sigma$ be two domains separated by a hypersurface $\bar{\Delta} \subset \Sigma \times \Sigma$ which contain the diagonal $\Delta=\{(u, u) \mid u \in \Sigma\} \subset \bar{\Delta}$. Assume that there exist distributions $G^{+}(u, z)$ and 
$G^{-}(u, z)$ regular in $\Omega^{+}$and $\Omega^{-}$respectively such that $\delta(u, z)=G^{+}(u, z)-G^{-}(u, z)$. To define half-currents corresponding to these Green distributions we decompose them as $G^{+}(u, z)=$ $\sum_{i} \alpha_{i}^{+}(u) \beta_{i}^{+}(z)$ and $G^{-}(u, z)=\sum_{i} \alpha_{i}^{-}(u) \beta_{i}^{-}(z)$. Then the half-currents are defined as $x^{+}(u)=$ $\sum_{i} \alpha_{i}^{+}(u) \hat{x}\left[\beta_{i}^{+}\right]$and $x^{-}(u)=\sum_{i} \alpha_{i}^{-}(u) \hat{x}\left[\beta_{i}^{-}\right]$. This definition does not depend on a choice of decompositions of the Green distributions. The half-currents are currents corresponding to the operators $\hat{x}^{ \pm}= \pm \hat{x} \cdot P^{ \pm}$, where $P^{ \pm}[s](z)= \pm\left\langle G^{ \pm}(u, z), s(u)\right\rangle, s \in \mathfrak{K}$. One can express the half-currents through the current $x(u)$, which we shall call a total current in contrast with the half ones:

$$
x^{+}(u)=\left\langle G^{+}(u, z) x(z)\right\rangle_{z}, \quad x^{-}(u)=\left\langle G^{-}(u, z) x(z)\right\rangle_{z}
$$

Here $\langle a(z)\rangle_{z} \equiv\langle a(z), 1\rangle_{z}$.

When $\Sigma$ is a covering of an elliptic curve we showed in [14] that:

- there are two essentially different choices of the test function algebras $\mathfrak{K}$ in this case corresponding to the different coverings $\Sigma$;

- the same quasi-doubly periodic meromorphic functions regularized with respect to the different test function algebras define the different quasi-Lie bialgebra structures and, therefore, the different classical elliptic current algebras;

- the internal structure of these two elliptic algebras is essentially different in spite of a similarity in the commutation relations between their half-currents.

Let $\tau \in \mathbb{C}, \operatorname{Im} \tau>0$ be a module of the elliptic curve $\mathbb{C} / \Gamma$, where $\Gamma=\mathbb{Z}+\tau \mathbb{Z}$ is a periodic lattice. The odd theta function $\theta(u)=-\theta(-u)$ is defined as a holomorphic function on $\mathbb{C}$ with the properties

$$
\theta(u+1)=-\theta(u), \quad \theta(u+\tau)=-e^{-2 \pi i u-\pi i \tau} \theta(u), \quad \theta^{\prime}(0)=1
$$

The first choice of the test function algebra corresponds to $\mathfrak{K}=\mathcal{K}_{0}$, where $\mathcal{K}_{0}$ consists of complex-valued one-variable functions defined in a vicinity of origin (see details in Appendix 4) equipped with the scalar product (4.1). These functions can be extended up to meromorphic functions on the covering $\Sigma=\mathbb{C}$. The regularization domains $\Omega^{+}, \Omega^{-}$for Green distributions in this case consist of the pairs $(u, z)$ such that $\max (1,|\tau|)>|u|>|z|>0$ and $0<|u|<|z|<$ $\max (1,|\tau|)$ respectively, where $\tau$ is an elliptic module, and $\bar{\Delta}=\{(u, z)|| u|=| z \mid\}$. We denote the corresponding elliptic Green distributions as $G_{\lambda}^{ \pm}(u, z)$ and $G(u, z)$, and their action on a test function $s(u)$ is defined as

$$
\begin{aligned}
\left\langle G_{\lambda}^{ \pm}(u, z), s(u)\right\rangle_{u} & =\oint_{\substack{|u|>|z| \\
|u|<|z|}} \frac{d u}{2 \pi i} \frac{\theta(u-z+\lambda)}{\theta(u-z) \theta(\lambda)} s(u) \\
\langle G(u, z), s(u)\rangle_{u} & =\oint_{|u|>|z|} \frac{d u}{2 \pi i} \frac{\theta^{\prime}(u-z)}{\theta(u-z)} s(u)
\end{aligned}
$$

where integrations are taken over circles around zero which are small enough such that the corresponding inequality takes place. The Green distributions are examples of the 'shifted' distributions (see Appendix 4) satisfying

$$
G_{\lambda}^{+}(u, z)-G_{\lambda}^{-}(u, z)=\delta(u, z), \quad G(u, z)+G(z, u)=\delta(u, z)
$$

The oddness of function $\theta(u)$ leads to the following connection between the $\lambda$-depending Green distributions: $G_{\lambda}^{+}(u, z)=-G_{-\lambda}^{-}(z, u)$. 
The second choice of the test function algebra corresponds to $\mathfrak{K}=K=K(\mathrm{Cyl})$. The algebra $K$ consists of entire periodic functions $s(u)=s(u+1)$ on $\mathbb{C}$ decaying exponentially at $\operatorname{Im} u \rightarrow \pm \infty$ equipped with an invariant scalar product (4.4). This functions can be regarded as functions on cylinder $\Sigma=\mathrm{Cyl}$ (see Appendix 4). The regularization domains $\Omega^{+}, \Omega^{-}$for Green distributions consist of the pairs $(u, z)$ such that $-\operatorname{Im} \tau<\operatorname{Im}(u-z)<0$ and $0<\operatorname{Im}(u-z)<\operatorname{Im} \tau$, respectively, and $\bar{\Delta}=\{(u, z) \mid \operatorname{Im} u=\operatorname{Im} z\}$. We denote the corresponding distributions as $\mathcal{G}_{\lambda}^{ \pm}(u-z)$ and $\mathcal{G}(u-z)$, and their action on the space $K$ is given by the formulas

$$
\begin{aligned}
\left\langle\mathcal{G}_{\lambda}^{ \pm}(u-z), s(u)\right\rangle_{u} & =\int_{\substack{-\operatorname{Im} \tau<\operatorname{Im}(u-z)<0 \\
0<\operatorname{Im}(u-z)<\operatorname{Im} \tau}} \frac{d u}{2 \pi i} \frac{\theta(u-z+\lambda)}{\theta(u-z) \theta(\lambda)} s(u) \\
\langle\mathcal{G}(u-z), s(u)\rangle_{u}= & \int_{-\operatorname{Im} \tau<\operatorname{Im}(u-z)<0} \frac{d u}{2 \pi i} \frac{\theta^{\prime}(u-z)}{\theta(u-z)} s(u)
\end{aligned}
$$

where the integration goes over the line segments of unit length (cycles of cylinder) such that the corresponding inequality takes place. The role of dual bases in the algebra $K$ is played by $\left\{j_{n}(u)=e^{2 \pi i n u}\right\}_{n \in \mathbb{Z}}$ and $\left\{j^{n}(u)=2 \pi i e^{-2 \pi i n u}\right\}_{n \in \mathbb{Z}}$ (see Appendix 4), a decomposition to these bases is the usual Fourier expansion. The Fourier expansions for the Green distributions are

$$
\mathcal{G}_{\lambda}^{ \pm}(u-z)= \pm 2 \pi i \sum_{n \in \mathbb{Z}} \frac{e^{-2 \pi i n(u-z)}}{1-e^{ \pm 2 \pi i(n \tau-\lambda)}}, \quad \mathcal{G}(u-z)=\pi i+2 \pi i \sum_{n \neq 0} \frac{e^{-2 \pi i n(u-z)}}{1-e^{2 \pi i n \tau}}
$$

These expansions are in accordance with formulae

$$
\mathcal{G}_{\lambda}^{+}(u-z)-\mathcal{G}_{\lambda}^{-}(u-z)=\delta(u-z), \quad \mathcal{G}(u-z)+\mathcal{G}(z-u)=\delta(u-z)
$$

where $\delta(u-z)$ is a delta-function on $K$, given by the expansion (4.5).

Using these two types of distributions we define in [14] two different quasi-Lie bialgebras $\mathfrak{e}_{\tau}\left(\widehat{\mathfrak{s l}}_{2}\right)$ and $\mathfrak{u}_{\tau}\left(\widehat{\mathfrak{s l}}_{2}\right)$, which are classical limits of quasi-Hopf algebras $E_{\tau, \eta}[2]$ and $U_{p, q}\left(\hat{\mathfrak{s l}}_{2}\right)$ [12] respectively. The algebraic and coalgebraic structures of these quasi-Lie bialgebras were described in terms of $L$-operators and classical $r$-matrices. In case of the algebra $\mathfrak{e}_{\tau}\left(\widehat{\mathfrak{s l}}_{2}\right)$ these objects are

$$
\begin{aligned}
L_{\lambda}^{ \pm}(u) & =\left(\begin{array}{ccc}
\frac{1}{2} h^{ \pm}(u) & f_{\lambda}^{ \pm}(u) \\
e_{\lambda}^{ \pm}(u) & -\frac{1}{2} h^{ \pm}(u)
\end{array}\right) \\
r_{\lambda}^{+}(u, v) & =\left(\begin{array}{cccc}
\frac{1}{2} G(u, v) & 0 & 0 & 0 \\
0 & -\frac{1}{2} G(u, v) & G_{-\lambda}^{+}(u, v) & 0 \\
0 & G_{\lambda}^{+}(u, v) & -\frac{1}{2} G(u, v) & 0 \\
0 & 0 & 0 & \frac{1}{2} G(u, v)
\end{array}\right)
\end{aligned}
$$

and can be obtained from the corresponding classical universal $r$-matrix using evaluation map. We denote the same objects in case of the algebra $\mathfrak{u}_{\tau}\left(\widehat{\mathfrak{s l}}_{2}\right)$ as $\mathcal{L}_{\lambda}^{ \pm}(u)$ and $r$-matrix $\mathfrak{r}_{\lambda}^{+}(u-v)$ with distributions $G(u, v)$ and $G_{\lambda}^{ \pm}(u, v)$ replaced everywhere by the distributions $\mathcal{G}(u, v)$ and $\mathcal{G}_{\lambda}^{ \pm}(u, v)$.

In Section 2 we describe different degenerations of the classical elliptic current algebras $\mathfrak{e}_{\tau}\left(\widehat{\mathfrak{s l}}_{2}\right)$ and $\mathfrak{u}_{\tau}\left(\widehat{\mathfrak{s l}}_{2}\right)$ in terms of degenerations of Green distributions entering the $r$-matrix. The degenerate Green distributions define the $r L L$-relations, the bialgebra structure and the analytic structure of half-currents. We do not write out explicitly the bialgebra structure related to the degenerated half-currents: it can be reconstructed along the lines of the paper [14].

We discuss the inverse problem in Section 3. A way to present the trigonometric and elliptic solutions of a Classical Yang-Baxter Equation (CYBE) by averaging of the rational ones was 
introduced in [4]. Faddeev and Reshetikhin applied the averaging method to a description of corresponding algebras. Here, we only represent the elliptic $r$-matrix $\mathfrak{r}_{\lambda}^{+}(u-v)$ and the trigonometric $r$-matrix $\mathfrak{r}^{(c)+}(u-v)$ as an average of the trigonometric $r$-matrix $\mathfrak{r}^{(b)+}(u-v)$ and the rational $r$-matrix $\mathfrak{r}^{(a)+}(u-v)$ respectively, for some domains of parameters.

Finally, in the Appendix, we have collected technical and "folklore" definitions and results concerning the test and distribution algebras on Riemman surfaces. Although some of the results can be extracted from standard textbooks $[7,15]$, we were not able to find them in the literature in the form suited for our goals and we have decided to keep them for the sake of completeness.

\section{Degenerated classical elliptic algebras}

We will describe a behavior of our algebras while one or both periods of the elliptic curve become infinite. The corresponding 'degenerated' Green distributions, $r$-matrix and $L$-operators give us a classical rational or a classical trigonometric 'limit' of corresponding elliptic current algebras.

\subsection{Degenerations of the quasi-Lie bialgebra $\mathfrak{e}_{\tau}\left(\widehat{\mathfrak{s l}}_{2}\right)$}

There are two different degenerations denoted (a) and (b) for $\mathfrak{e}_{\tau}\left(\widehat{\mathfrak{s l}}_{2}\right)$. (a) corresponds to the case when both periods are infinite $\left(\omega \rightarrow \infty, \omega^{\prime} \rightarrow \infty\right)$. This is a rational degeneration. In the case (b) one of the periods is infinite $\left(\omega^{\prime} \rightarrow \infty\right)$ while another $(\omega)$ rests finite. This is a case of trigonometric degeneration. A situation when $\omega \rightarrow \infty$ and $\omega^{\prime}$ is finite, is equivalent to (b) due to the symmetry of integration contour and, therefore, we do not consider it separately.

2.1.1. Case $(\mathbf{a}): \omega \rightarrow \infty, \omega^{\prime} \rightarrow \infty,\left(\operatorname{Im} \frac{\omega^{\prime}}{\omega}>0\right)$. In order to turn to the lattice of periods $\Gamma=\mathbb{Z} \omega+\mathbb{Z} \omega^{\prime}$, with $\frac{\omega^{\prime}}{\omega}=\tau$, we need to re-scale the variables like $u \rightarrow \frac{u}{\omega}$. Let us introduce the following notations for rational Green distributions

$$
\left\langle\varphi^{ \pm}(u, z), s(u)\right\rangle_{u}=\oint_{\substack{|u|>|z| \\|u|<|z|}} \frac{d u}{2 \pi i} \frac{1}{u-z} s(u)
$$

These distributions are degenerations of elliptic Green distributions:

$$
\frac{1}{\omega} G\left(\frac{u}{\omega}, \frac{z}{\omega}\right) \rightarrow \varphi^{+}(u, z), \quad \frac{1}{\omega} G_{\frac{\lambda}{\omega}}^{ \pm}\left(\frac{u}{\omega}, \frac{z}{\omega}\right) \rightarrow \frac{1}{\lambda}+\varphi^{ \pm}(u, z)
$$

and the $r$-matrix tends to

$$
\begin{aligned}
r_{\lambda}^{(a)+}(u, v) & =\lim _{\omega, \omega^{\prime} \rightarrow \infty} \frac{1}{\omega} r_{\frac{\lambda}{\omega}}^{+}\left(\frac{u}{\omega}, \frac{v}{\omega}\right)= \\
& =\left(\begin{array}{cccc}
\frac{1}{2} \varphi^{+}(u, v) & 0 & 0 & 0 \\
0 & -\frac{1}{2} \varphi^{+}(u, v) & -\frac{1}{\lambda}+\varphi^{+}(u, v) & 0 \\
0 & \frac{1}{\lambda}+\varphi^{+}(u, v) & -\frac{1}{2} \varphi^{+}(u, v) & 0 \\
0 & 0 & 0 & \frac{1}{2} \varphi^{+}(u, v)
\end{array}\right)
\end{aligned}
$$

Actually the quasi-Lie bialgebras obtained as rational degenerations of $\mathfrak{e}_{\tau}\left(\widehat{\mathfrak{s l}}_{2}\right)$ for different $\lambda$ are related to each other by very simple twist. Therefore we shall consider only one value of the parameter $\lambda$, namely we shall consider the limit value $\lambda \rightarrow \infty$. The $r$-matrix and $L$-operators look then as follows

$$
\begin{aligned}
L^{(a) \pm}(u) & =\lim _{\lambda \rightarrow \infty} \lim _{\omega, \omega^{\prime} \rightarrow \infty} \frac{1}{\omega} L_{\frac{\lambda}{\omega}}^{ \pm}\left(\frac{u}{\omega}\right)=\left(\begin{array}{cc}
\frac{1}{2} h^{(a) \pm}(u) & f^{(a) \pm}(u) \\
e^{(a) \pm}(u) & -\frac{1}{2} h^{(a) \pm}(u)
\end{array}\right) \\
r^{(a)+}(u, v) & =\lim _{\lambda \rightarrow \infty} \frac{1}{\omega} r_{\lambda}^{(a)+}(u, v)
\end{aligned}
$$




$$
=\left(\begin{array}{cccc}
\frac{1}{2} \varphi^{+}(u, v) & 0 & 0 & 0 \\
0 & -\frac{1}{2} \varphi^{+}(u, v) & \varphi^{+}(u, v) & 0 \\
0 & \varphi^{+}(u, v) & -\frac{1}{2} \varphi^{+}(u, v) & 0 \\
0 & 0 & 0 & \frac{1}{2} \varphi^{+}(u, v)
\end{array}\right)
$$

Substituting $u \rightarrow \frac{u}{\omega}, v \rightarrow \frac{v}{\omega}, \lambda \rightarrow \frac{\lambda}{\omega}$ into commutation relations of the algebra $\mathfrak{e}_{\tau}\left(\widehat{\mathfrak{s l}}_{2}\right)$ given by the formulas (3.38) and (3.39) of the paper [14], multiplying it by $\frac{1}{\omega^{2}}$ and passing to the limits we obtain

$$
\begin{aligned}
{\left[L_{1}^{(a), \pm}(u), L_{2}^{(a) \pm}(v)\right] } & =\left[L_{1}^{(a) \pm}(u)+L_{2}^{(a) \pm}(v), r^{(a)+}(u, v)\right] \\
{\left[L_{1}^{(a)+}(u), L_{2}^{(a)-}(v)\right] } & =\left[L_{1}^{(a)+}(u)+L_{2}^{(a)-}(v), r^{(a)+}(u, v)\right]+c \cdot \frac{\partial}{\partial u} r^{(a)+}(u, v)
\end{aligned}
$$

The half-currents have decompositions

$$
x^{(a)+}(u)=\sum_{n \geq 0} x_{n}^{(a)} u^{-n-1}, \quad x^{(a)-}(u)=-\sum_{n<0} x_{n}^{(a)} u^{-n-1}
$$

where $x_{n}^{(a)}=\left(x \otimes z^{n}, 0,0\right)$ for $n \in \mathbb{Z}, x \in\{h, e, f\}$. This means that this algebra coincides with a classical limit of the central extension of the Yangian double $\widehat{D Y\left(\mathfrak{s l}_{2}\right)}[8]$.

2.1.2. Case (b): $\tau \rightarrow i \infty\left(\omega=1, \omega^{\prime} \rightarrow \infty, \tau=\omega^{\prime} / \omega, \operatorname{Im} \tau>0\right)$. In this case the degenerations of elliptic Green distributions look as follows:

$$
\begin{aligned}
G(u, z) & \rightarrow \psi^{+}(u-z) \\
G_{\lambda}^{ \pm}(u, z) & \rightarrow \pi \operatorname{ctg} \pi \lambda+\psi^{ \pm}(u, z)
\end{aligned}
$$

where

$$
\left\langle\psi^{ \pm}(u, z), s(u)\right\rangle_{u}=\oint_{\substack{|u|>|z| \\|u|<|z|}} \frac{d u}{2 \pi i} \pi \operatorname{ctg} \pi(u-z) s(u)
$$

By the same reason the degenerated algebras are isomorphic for different $\lambda$, and we shall consider this bialgebra only in the limit $\lambda \rightarrow-i \infty$. The $r$-matrix, $L$-operators and $r L L$-relations in this case take the form

$$
\begin{aligned}
& r^{(b)+}(u, v)=\left(\begin{array}{cccc}
\frac{1}{2} \psi^{+}(u, v) & 0 & 0 & 0 \\
0 & -\frac{1}{2} \psi^{+}(u, v) & -\pi i+\psi^{+}(u, v) & 0 \\
0 & \pi i+\psi^{+}(u, v) & -\frac{1}{2} \psi^{+}(u, v) & 0 \\
0 & 0 & 0 & \frac{1}{2} \psi^{+}(u, v)
\end{array}\right) \\
& L^{(b) \pm}(u)=\lim _{\lambda \rightarrow-i \infty} \lim _{\tau \rightarrow i \infty} L_{\lambda}^{ \pm}(u)=\left(\begin{array}{cc}
\frac{1}{2} h^{(b) \pm}(u) & f^{(b) \pm}(u) \\
e^{(b) \pm}(u) & -\frac{1}{2} h^{(b) \pm}(u)
\end{array}\right) \\
& {\left[L_{1}^{(b) \pm}(u), L_{2}^{(b) \pm}(v)\right]=\left[L_{1}^{(b) \pm}(u)+L_{2}^{(b) \pm}(v), r^{(b)+}(u, v)\right]} \\
& {\left[L_{1}^{(b)+}(u), L_{2}^{(b)-}(v)\right]=\left[L_{1}^{(b)+}(u)+L_{2}^{(b)-}(v), r^{(b)+}(u, v)\right]+c \cdot \frac{\partial}{\partial u} r^{(b)+}(u, v)}
\end{aligned}
$$

The half-currents have the following decompositions

$$
\begin{aligned}
h^{(b)+}(u) & =\sum_{n \geq 0} h_{n}^{(b)} \frac{\partial^{n}}{\partial u^{n}} \operatorname{ctg} \pi u, & h^{(b)-}(u) & =-\sum_{n \geq 0} h_{-n-1}^{(b)} u^{n} \\
e^{(b)+}(u) & =i e_{0}^{(b)}+\sum_{n \geq 0} e_{n}^{(b)} \frac{\partial^{n}}{\partial u^{n}} \operatorname{ctg} \pi u, & e^{(b)-}(u) & =i e_{0}^{(b)}-\sum_{n \geq 0} e_{-n-1}^{(b)} u^{n}
\end{aligned}
$$




$$
f^{(b)+}(u)=-i f_{0}^{(b)}+\sum_{n \geq 0} f_{n}^{(b)} \frac{\partial^{n}}{\partial u^{n}} \operatorname{ctg} \pi u, \quad f^{(b)-}(u)=-i f_{0}^{(b)}-\sum_{n \geq 0} f_{-n-1}^{(b)} u^{n}
$$

where

$$
x_{n}^{(b)}=\left(x \otimes \pi \frac{(-1)^{n}}{n !} z^{n}, 0,0\right), \quad x_{-n-1}^{(b)}=\left(x \otimes \pi \frac{(-1)^{n}}{n !} \frac{\partial^{n}}{\partial z^{n}} \operatorname{ctg} \pi z, 0,0\right)
$$

for $n \geq 0, x \in \mathfrak{s l}_{2}$.

\subsection{Degeneration of the quasi-Lie bialgebra $\mathfrak{u}_{\tau}\left(\widehat{\mathfrak{s l}}_{2}\right)$}

In the case of algebra $\mathfrak{u}_{\tau}\left(\widehat{\mathfrak{s l}}_{2}\right)$ there are three cases of degenerations: (a), (b) and (c). The rational degeneration (a) and trigonometric degeneration (b) are analogous to the corresponding degenerations of $\mathfrak{e}_{\tau}\left(\widehat{\mathfrak{s l}}_{2}\right)$. Additionally there is one more trigonometric case $(\mathbf{c})$, when $\omega \rightarrow \infty$ and $\omega^{\prime}$ is finite. It is not equivalent to the case (b) because the integration contour for $\mathfrak{u}_{\tau}\left(\widehat{\mathfrak{s l}}_{2}\right)$ is not symmetric in this case. In the cases (a) and (c) the degeneration of elliptic Green distributions acts on another test function algebra $Z$. This is an algebra of entire functions $s(u)$ subject to the inequalities $\left|u^{n} s(u)\right|<C_{n} e^{p|\operatorname{Im} u|}, n \in \mathbb{Z}_{+}$, for some constants $C_{n}, p>0$ depending on $s(u)$ [7]. The scalar product in $Z$ is $\langle s(u), t(u)\rangle_{u}=\int_{-\infty}^{+\infty} \frac{d u}{2 \pi i} s(u) t(u)$. The distributions acting on $K$ can be considered as periodic distributions acting on $Z$.

2.2.1. Case $(\mathbf{a}): \omega \rightarrow \infty, \omega^{\prime} \rightarrow \infty,\left(\operatorname{Im} \frac{\omega^{\prime}}{\omega}>0\right)$. The degenerating of the elliptic Green distributions in this case reads as

$$
\begin{aligned}
\frac{1}{\omega} \mathcal{G}\left(\frac{u-z}{\omega}\right) & \rightarrow \frac{1}{u-z-i 0}=\Phi^{+}(u-z) \\
\frac{1}{\omega} \mathcal{G}_{\frac{\lambda}{\omega}}^{ \pm}\left(\frac{u-z}{\omega}\right) & \rightarrow \frac{u-z+\lambda}{(u-z \mp i 0) \lambda}=\frac{1}{\lambda}+\Phi^{ \pm}(u-z)
\end{aligned}
$$

where we introduced the rational Green distributions acting on the test function algebra $Z$ by formula

$$
\left\langle\Phi^{ \pm}(u-z), s(u)\right\rangle=\int_{\substack{\operatorname{Im} u<\operatorname{Im} z \\ \operatorname{Im} u>\operatorname{Im} z}} \frac{d u}{2 \pi i} \frac{1}{u-z} s(u)
$$

with infinite horizontal integration lines. They can be represented as integrals

$$
\begin{aligned}
& \Phi^{+}(u-z)=2 \pi i \int_{-\infty}^{0} e^{2 \pi i k(u-z)} d k=2 \pi i \int_{0}^{+\infty} e^{-2 \pi i k(u-z)} d k \\
& \Phi^{-}(u-z)=-2 \pi i \int_{0}^{+\infty} e^{2 \pi i k(u-z)} d k=-2 \pi i \int_{-\infty}^{0} e^{-2 \pi i k(u-z)} d k
\end{aligned}
$$

These formulae are degenerations of the expansions (1.8).

As above, all the algebras that are obtained from the limit $\omega, \omega^{\prime} \rightarrow \infty$ are isomorphic for the different values of the parameter $\lambda$ and it is sufficient to describe the limit case $\lambda=\infty$. The $r L L$-relations are the same as $(2.2),(2.3)$

$$
\begin{aligned}
{\left[\mathcal{L}_{1}^{(a), \pm}(u), \mathcal{L}_{2}^{(a) \pm}(v)\right] } & =\left[\mathcal{L}_{1}^{(a) \pm}(u)+\mathcal{L}_{2}^{(a) \pm}(v), \mathfrak{r}^{(a)+}(u-v)\right] \\
{\left[\mathcal{L}_{1}^{(a)+}(u), \mathcal{L}_{2}^{(a)-}(v)\right] } & =\left[\mathcal{L}_{1}^{(a)+}(u)+\mathcal{L}_{2}^{(a)-}(v), \mathfrak{r}^{(a)+}(u-v)\right]+c \cdot \frac{\partial}{\partial u} \mathfrak{r}^{(a)+}(u-v)
\end{aligned}
$$


with similar $r$-matrix

$$
\begin{aligned}
\mathfrak{r}^{(a)+}(u-v) & =\lim _{\lambda \rightarrow \infty} \lim _{\omega, \omega^{\prime} \rightarrow \infty} \frac{1}{\omega} \mathfrak{r}_{\frac{\lambda}{\omega}}^{+}\left(\frac{u-v}{\omega}\right)= \\
& =\left(\begin{array}{cccc}
\frac{1}{2} \Phi^{+}(u-v) & 0 & 0 & 0 \\
0 & -\frac{1}{2} \Phi^{+}(u-v) & \Phi^{+}(u-v) & 0 \\
0 & \Phi^{+}(u-v) & -\frac{1}{2} \Phi^{+}(u-v) & 0 \\
0 & 0 & 0 & \frac{1}{2} \Phi^{+}(u-v)
\end{array}\right)
\end{aligned}
$$

but the entries of the $L$-matrix

$$
\mathcal{L}^{(a) \pm}(u)=\lim _{\lambda \rightarrow \infty} \lim _{\omega, \omega^{\prime} \rightarrow \infty} \frac{1}{\omega} \mathcal{L}_{\frac{\lambda}{\omega}}^{ \pm}\left(\frac{u}{\omega}\right)=\left(\begin{array}{cc}
\frac{1}{2} h^{(a) \pm}(u) & f^{(a) \pm}(u) \\
e^{(a) \pm}(u) & -\frac{1}{2} h^{(a) \pm}(u)
\end{array}\right)
$$

are decomposed to the integrals instead of the series:

$$
x^{(a)+}(u)=\int_{0}^{+\infty} x_{k}^{(a)} e^{-2 \pi i k u} d k, \quad x^{(a)-}(u)=-\int_{-\infty}^{0} x_{k}^{(a)} e^{-2 \pi i k u} d k
$$

where $x_{k}^{(a)}=\left(x \otimes 2 \pi i e^{2 \pi i k z}, 0,0\right), x \in\{h, e, f\}$. These half-currents form a quasi-classical degeneration of the algebra $\mathcal{A}_{\hbar}\left(\widehat{\mathfrak{s l}}_{2}\right)$ (see [10]). The difference between algebras $\widehat{D Y\left(\mathfrak{s l}_{2}\right)}$ and $\mathcal{A}_{\hbar}\left(\widehat{\mathfrak{s l}_{2}}\right)$ is considered in details on the quantum level in this paper.

2.2.2. Case (b). $\tau \rightarrow i \infty,\left(\omega=1, \omega^{\prime} \rightarrow \infty, \tau=\omega^{\prime} / \omega, \operatorname{Im} \tau>0\right)$. Taking the limit $\tau \rightarrow i \infty$ in the formula (1.8) we obtain

$$
\begin{gathered}
\mathcal{G}(u-z) \rightarrow \pi i+2 \pi i \sum_{n>0} e^{-2 \pi i n(u-z)}=\tilde{\psi}^{+}(u-z) \\
\mathcal{G}_{\lambda}^{ \pm}(u-z) \rightarrow \pi \operatorname{ctg} \pi \lambda-\pi i \pm 2 \pi i \sum_{\substack{n \geq 0 \\
n<0}} e^{-2 \pi i n(u-z)}=\pi \operatorname{ctg} \pi \lambda+\tilde{\psi}^{ \pm}(u-z)
\end{gathered}
$$

where

$$
\left\langle\tilde{\psi}^{ \pm}(u-z), s(u)\right\rangle=\int_{\substack{\operatorname{Im} u<\operatorname{Im} z \\ \operatorname{Im} u>\operatorname{Im} z}} \frac{d u}{2 \pi i} \pi \operatorname{ctg} \pi(u-z) s(u)
$$

where $s \in K$ and the integration is taken over a horizontal line segments with unit length. In these notations the $r$-matrix (in the limit $\lambda \rightarrow-i \infty$ ) can be written as

$$
\begin{aligned}
\mathfrak{r}^{(b)+}(u-v) & =\lim _{\lambda \rightarrow-i \infty} \lim _{\tau \rightarrow i \infty} \mathfrak{r}_{\lambda}^{+}(u-v) \\
& =\left(\begin{array}{cccc}
\frac{1}{2} \tilde{\psi}^{+}(u-v) & 0 & 0 & 0 \\
0 & -\frac{1}{2} \tilde{\psi}^{+}(u-v) & -\pi i+\tilde{\psi}^{+}(u-v) & 0 \\
0 & \pi i+\tilde{\psi}^{+}(u-v) & -\frac{1}{2} \tilde{\psi}^{+}(u-v) & 0 \\
0 & 0 & 0 & \frac{1}{2} \tilde{\psi}^{+}(u-v)
\end{array}\right)
\end{aligned}
$$

Setting

$$
\mathcal{L}^{(b) \pm}(u)=\lim _{\lambda \rightarrow-i \infty} \lim _{\tau \rightarrow i \infty} \mathcal{L}_{\lambda}^{ \pm}(u)=\left(\begin{array}{cc}
\frac{1}{2} h^{(b) \pm}(u) & f^{(b) \pm}(u) \\
e^{(b) \pm}(u) & -\frac{1}{2} h^{(b) \pm}(u)
\end{array}\right)
$$

one derives

$$
\left[\mathcal{L}_{1}^{(b), \pm}(u), \mathcal{L}_{2}^{(b) \pm}(v)\right]=\left[\mathcal{L}_{1}^{(b) \pm}(u)+\mathcal{L}_{2}^{(b) \pm}(v), \mathfrak{r}^{(b)+}(u-v)\right]
$$




$$
\left[\mathcal{L}_{1}^{(b)+}(u), \mathcal{L}_{2}^{(b)-}(v)\right]=\left[\mathcal{L}_{1}^{(b)+}(u)+\mathcal{L}_{2}^{(b)-}(v), \mathfrak{r}^{(b)+}(u-v)\right]+c \cdot \frac{\partial}{\partial u} \mathfrak{r}^{(b)+}(u-v)
$$

which define some Lie algebra together with half-current decompositions

$$
\begin{aligned}
h^{(b)+}(u) & =-\frac{1}{2} h_{0}^{(a)}+\sum_{n \geq 0} h_{n}^{(a)} e^{-2 \pi i n u}, & h^{(b)-}(u) & =-\frac{1}{2} h_{0}^{(a)}-\sum_{n<0} h_{n}^{(a)} e^{-2 \pi i n u} \\
e^{(b)+}(u) & =\sum_{n \geq 0} e_{n}^{(a)} e^{-2 \pi i n u}, & e^{(b)-}(u) & =-\sum_{n<0} e_{n}^{(a)} e^{-2 \pi i n u} \\
f^{(b)+}(u) & =\sum_{n>0} f_{n}^{(a)} e^{-2 \pi i n u}, & f^{(b)-}(u) & =-\sum_{n \leq 0} f_{n}^{(a)} e^{-2 \pi i n u}
\end{aligned}
$$

where $x_{k}^{(b)}=\left(x \otimes 2 \pi i e^{2 \pi i n z}, 0,0\right), x \in\{h, e, f\}$. This is exactly an affine Lie algebra $\widehat{\mathfrak{s l}_{2}}$ with a bialgebra structure inherited from the quantum affine algebra $U_{q}\left(\widehat{\mathfrak{s l}_{2}}\right)$.

2.2.3. Case (c): $\omega=\rightarrow \infty, \omega^{\prime}=\tau \omega=\frac{i}{\eta}=$ const, $(\operatorname{Re} \eta>0)$. Substituting $u \rightarrow \frac{u}{\omega}, z \rightarrow \frac{z}{\omega}$ to the expansion for $\mathcal{G}(u-z)$ we yield the following degeneration

$$
\frac{1}{\omega} \mathcal{G}\left(\frac{u-z}{\omega}\right) \rightarrow \Psi(u-z) \stackrel{\text { def }}{=} 2 \pi i \int_{-\infty}^{+\infty} \frac{e^{-2 \pi i k(u-z)} d k}{1-e^{-\frac{2 \pi k}{\eta}}}
$$

Here $f$ means an integral in sense of principal value. The integral in the formula (2.8) converges in the domain $\operatorname{Re} \eta^{-1}<\operatorname{Im}(u-z)<0$ and is equal to $\pi \eta \operatorname{cth} \pi \eta(u-z)$ in this domain. It means that the distribution $\Psi(u-z)$ defined by formula (2.8) acts on $Z$ as follows

$$
\langle\Psi(u-z), s(u)\rangle=\int_{-\operatorname{Re} \eta^{-1}<\operatorname{Im}(u-z)<0} \frac{d u}{2 \pi i} \pi \eta \operatorname{cth} \pi \eta(u-z) s(u)
$$

The degeneration of Green distributions parameterized by $\lambda$ can be performed in different ways. We can consider a more general substitution $\lambda \rightarrow \mu+\frac{\lambda}{\omega}$ instead of $\lambda \rightarrow \frac{\lambda}{\omega}$ used above. Substituting $u \rightarrow \frac{u}{\omega}, z \rightarrow \frac{z}{\omega}, \lambda \rightarrow \mu+\frac{\lambda}{\omega}$ to the formula (1.8) and taking the limits $\omega \rightarrow \infty$ and $\lambda \rightarrow \infty$ we obtain

$$
\begin{gathered}
\frac{1}{\omega} \mathcal{G}_{\mu+\frac{\lambda}{\omega}}^{+}\left(\frac{u-z}{\omega}\right) \rightarrow 2 \pi i \int_{-\infty}^{\infty} \frac{e^{-2 \pi i k(u-z)} d k}{1-e^{-\frac{2 \pi k}{\eta}-2 \pi i \mu}} \\
\frac{1}{\omega} \mathcal{G}_{\mu+\frac{\lambda}{\omega}}^{-}\left(\frac{u-z}{\omega}\right) \rightarrow 2 \pi i \int_{-\infty}^{\infty} \frac{e^{-2 \pi i k(u-z)} d k}{e^{\frac{2 \pi k}{\eta}+2 \pi i \mu}-1}
\end{gathered}
$$

Left hand sides of (2.10) and (2.11) as well as right hand sides are invariant under $\mu \rightarrow \mu+1$, but the right hand side is not holomorphic with respect to $\mu$ because of integrand poles. The complex plane split up to the following analyticity zones

$$
\frac{\operatorname{Im} \eta \operatorname{Im} \mu}{\operatorname{Re} \eta}+n<\operatorname{Re} \mu<\frac{\operatorname{Im} \eta \operatorname{Im} \mu}{\operatorname{Re} \eta}+n+1, \quad n \in \mathbb{Z}
$$

and due to periodicity with respect to $\mu$ one can consider only one of these zones.

Integrals in the formulae (2.10) and (2.11) converge in the domain $\operatorname{Re} \eta^{-1}<\operatorname{Im}(u-z)<0$ and $0<\operatorname{Im}(u-z)<\operatorname{Re} \eta^{-1}$ respectively and they can be calculated like the integral in (2.8) for the chosen zone. Denote by $\Psi_{\mu}^{+}(u-z)$ and $\Psi_{\mu}^{-}(u-z)$ the analytic continuation with respect to $\mu$ of the right hand sides of (2.10) and (2.11) respectively from the zone

$$
\frac{\operatorname{Im} \eta \operatorname{Im} \mu}{\operatorname{Re} \eta}<\operatorname{Re} \mu<\frac{\operatorname{Im} \eta \operatorname{Im} \mu}{\operatorname{Re} \eta}+1
$$


Thus, this degeneration of Green distributions can be rewritten as

$$
\begin{aligned}
\lim _{\omega \rightarrow \infty} \mathcal{G}_{\mu+\frac{\lambda}{\omega}}^{ \pm}\left(\frac{u-z}{\omega}\right)= & \Psi_{\mu}^{ \pm}(u-z), \\
\left\langle\Psi_{\mu}^{ \pm}(u-z), s(u)\right\rangle= & \int_{\substack{-\operatorname{Re} \eta^{-1}<\operatorname{Im}(u-z)<0 \\
0<\operatorname{Im}(u-z)<\operatorname{Re} \eta^{-1}}} \frac{d u}{2 \pi i} 2 \pi \eta \frac{e^{-2 \pi \eta \mu(u-z)}}{1-e^{-2 \pi \eta(u-z)}} s(u)
\end{aligned}
$$

where $s \in Z$ and the integrals are taken over the horizontal lines.

For the values For the values $\operatorname{Re} \mu=\frac{\operatorname{Im} \eta \operatorname{Im} \mu}{\operatorname{Re} \eta}$, integrands in $(2.10),(2.11)$ have a pole on the real axis and the distributions $\Psi_{\mu}^{+}(u-z)$ and $\Psi_{\mu}^{-}(u-z)$ regularize these integrals as analytical continuation (see [7]). The $r$-matrix obtained by another regularization does not satisfy the CYBE.

The degeneration of $r$-matrix is ${ }^{1}$

$$
\begin{aligned}
\mathfrak{r}^{(c)+}(u-v) & =\lim _{\lambda \rightarrow \infty} \lim _{\omega \rightarrow \infty} \frac{1}{\omega} \mathfrak{r}^{+}{ }_{\mu+\frac{\lambda}{\omega}}\left(\frac{u-v}{\omega}\right) \\
& =\left(\begin{array}{cccc}
\frac{1}{2} \Psi(u-v) & 0 & 0 & 0 \\
0 & -\frac{1}{2} \Psi(u-v) & -\Psi_{\mu}^{-}(v-u) & 0 \\
0 & \Psi_{\mu}^{+}(u-v) & -\frac{1}{2} \Psi(u-v) & 0 \\
0 & 0 & 0 & \frac{1}{2} \Psi(u-v)
\end{array}\right)
\end{aligned}
$$

The $L$-operators

$$
\mathcal{L}^{(c) \pm}(u)=\lim _{\lambda \rightarrow \infty} \lim _{\omega \rightarrow \infty} \frac{1}{\omega} \mathcal{L}_{\mu+\frac{\lambda}{\omega}}^{ \pm}\left(\frac{u}{\omega}\right)=\left(\begin{array}{cc}
\frac{1}{2} h^{(c) \pm}(u) & f^{(c) \pm}(u) \\
e^{(c) \pm}(u) & -\frac{1}{2} h^{(c) \pm}(u)
\end{array}\right)
$$

with this $r$-matrix satisfy the dynamical $r L L$-relations

$$
\begin{aligned}
{\left[\mathcal{L}_{1}^{(c) \pm}(u), \mathcal{L}_{2}^{(c) \pm}(v)\right] } & =\left[\mathcal{L}_{1}^{(c) \pm}(u)+\mathcal{L}_{2}^{(c) \pm}(v), \mathfrak{r}^{(c)+}(u-v)\right]-c \cdot i \eta^{2} \frac{\partial}{\partial \eta} \mathfrak{r}^{(c)+}(u-v) \\
{\left[\mathcal{L}_{1}^{(c)+}(u), \mathcal{L}_{2}^{(c)-}(v)\right] } & =\left[\mathcal{L}_{1}^{(c)+}(u)+\mathcal{L}_{2}^{(c)-}(v), \mathfrak{r}^{(c)+}(u-v)\right] \\
& +c \cdot\left(\frac{\partial}{\partial u}-i \eta^{2} \frac{\partial}{\partial \eta}\right) \mathfrak{r}^{(c)+}(u-v)
\end{aligned}
$$

Decompositions of the half-current in this degeneration are

$$
\begin{aligned}
h^{(c)+}(u) & =\int_{-\infty}^{+\infty} h_{k}^{(c)} \frac{e^{-2 \pi i k u} d k}{1-e^{-\frac{2 \pi k}{\eta}}}, & h^{(c)-}(u) & =\int_{-\infty}^{+\infty} h_{k}^{(c)} \frac{e^{-2 \pi i k u} d k}{e^{\frac{2 \pi k}{\eta}}-1} \\
e^{(c)+}(u) & =\int_{-\infty}^{+\infty} e_{k}^{(c)} \frac{e^{-2 \pi i k u} d k}{1-e^{-\frac{2 \pi k}{\eta}-2 \pi i \mu}}, & e^{(c)-}(u) & =\int_{-\infty}^{+\infty} e_{k}^{(c)} \frac{e^{-2 \pi i k u} d k}{e^{\frac{2 \pi k}{\eta}+2 \pi i \mu}-1} \\
f^{(c)+}(u)= & \int_{-\infty}^{+\infty} f_{k}^{(c)} \frac{e^{-2 \pi i k u} d k}{1-e^{-\frac{2 \pi k}{\eta}+2 \pi i \mu}}, & f^{(c)-}(u) & =\int_{-\infty}^{+\infty} f_{k}^{(c)} \frac{e^{-2 \pi i k u} d k}{e^{\frac{2 \pi k}{\eta}-2 \pi i \mu}-1}
\end{aligned}
$$

\footnotetext{
${ }^{1}$ Let us remark that the degeneration of the entry $r^{+}(u-v)_{12,21}=\mathcal{G}_{-\lambda}^{+}(u-v)$ in the zone (2.12) is $\Psi_{1-\mu}^{+}(u-v)$, but is not $\Psi_{-\mu}^{+}(u-v)$ as one could expect, because of periodicity with respect to $\mu$ and the fact that $\mu$ belongs to the zone (2.12) if and only if $1-\mu$ belongs to the zone (2.12). One can also use the relations $\mathcal{G}_{-\mu-\frac{\lambda}{\omega}}^{+}\left(\frac{u-v}{\omega}\right)=$ $-\mathcal{G}_{\mu+\frac{\lambda}{\omega}}^{-}\left(\frac{v-u}{\omega}\right) \longrightarrow-\Psi_{\mu}^{-}(v-u)=\Psi_{1-\mu}^{+}(u-v)$.
} 
where $x_{k}^{(a)}=x \otimes 2 \pi i e^{2 \pi i k z}, x \in\{h, e, f\}$. We do not make explicit the dependence on the parameter $\mu$ because, contrary to $\lambda$, it is not a dynamical parameter. We also omit dependence on the parameter $\eta$ which provides the dynamics over $c$ just as we omitted its analogue $\tau$ in the elliptic case. The case $\mu=\frac{1}{2}, \operatorname{Im} \eta=0$ coincides with the quasi-classical limit of the quantum current algebra $\mathcal{A}_{\hbar, \eta}\left(\widehat{\mathfrak{s l}_{2}}\right)[9,13]$. This algebra was investigated in [11] in detail. Other degenerations (c) seem to be unknown, though the matrices $\mathfrak{r}^{(c)+}(u)$ fit the Belavin-Drinfeld classification [1].

\section{Averaging of $r$-matrices}

Now we will use the averaging method of Faddeev-Reshetikhin [4] and will write down trigonometric and elliptic $r$-matrices starting with a rational and a trigonometric solution of CYBE respectively. We show that the $r$-matrices satisfying a Dynamical Classical Yang-Baxter Equation can be also obtained by this method.

CYBE. A meromorphic $\mathfrak{a} \otimes \mathfrak{a}$-valued function $X(u)$ (in our case $\mathfrak{a}=\mathfrak{s l}_{2}$ ) is called solution of the CYBE if it satisfies the equation

$$
\begin{aligned}
{\left[X_{12}\left(u_{1}-u_{2}\right), X_{13}\left(u_{1}-u_{3}\right)\right]+\left[X_{12}\left(u_{1}-u_{2}\right),\right.} & \left.X_{23}\left(u_{2}-u_{3}\right)\right]+ \\
& +\left[X_{13}\left(u_{1}-u_{3}\right), X_{23}\left(u_{2}-u_{3}\right)\right]=0
\end{aligned}
$$

The $r$-matrices $\mathfrak{r}^{(a)+}(u-v), \mathfrak{r}^{(b)+}(u-v), \mathfrak{r}^{(c)+}(u-v)$ defined by formulae (2.5), (2.7) and (2.14) satisfy CYBE (3.1). This follows from the fact that they are regularization of the corresponding rational and trigonometric solutions of $\mathrm{CYBE}$ in the domain $\operatorname{Im} u<\operatorname{Im} v$. Indeed, in order to check the equation (3.1) for these $r$-matrices it is sufficient to check it in the domain $\operatorname{Im} u_{1}<$ $\operatorname{Im} u_{2}<\operatorname{Im} u_{3}$. The regularization of the same first two solutions of CYBE ((a) and (b) cases) but in domain $|u|>|v|$ are $r$-matrices $\mathfrak{r}^{(a)+}(u-v)$ and $\mathfrak{r}^{(b)+}(u-v)$ (formulae (2.4) and (2.4)) respectively. Hence they also satisfy $(3.1)$, but with $X_{i j}\left(u_{i}-u_{j}\right)$ is replaced by $X_{i j}\left(u_{i}, u_{j}\right)$. The elliptic $r$-matrix $\mathfrak{r}_{\lambda}^{+}(u-v)$ satisfies Dynamical CYBE, but it can be also obtained by the averaging method.

Basis of averaging. As it was shown in [1] each solution of CYBE $X(u)$ is a rational, trigonometric or elliptic (doubly periodic) function of $u$, the poles of $X(u)$ form a lattice $\mathfrak{R} \subset \mathbb{C}$ and there is a group homomorphism $A: \mathfrak{R} \rightarrow$ Aut $\mathfrak{g}$ such that for each $\gamma \in \mathfrak{R}$ one has the relation $X(u+\gamma)=\left(A_{\gamma} \otimes \mathrm{id}\right) X(u)$. Having a rational solution $X(u)$, for which $\mathfrak{R}=\{0\}$, and choosing an appropriate automorphisms $A=A_{\gamma_{0}}$ we can construct the trigonometric solution with $\mathfrak{R}=\gamma_{0} \mathbb{Z}$ in the form

$$
\sum_{n \in \mathbb{Z}}\left(A^{n} \otimes \mathrm{id}\right) X\left(u-n \gamma_{0}\right)
$$

Applying the same procedure for a trigonometric solution with $\mathfrak{R}=\gamma_{1} \mathbb{Z}$, where $\gamma_{1} / \gamma_{0} \notin \mathbb{R}$, we obtain an elliptic (doubly periodic) solution of CYBE with $\mathfrak{R}=\gamma_{1} \mathbb{Z}+\gamma_{0} \mathbb{Z}$. The convergence of series in the formula (3.2) should be understood in the principal value sense (below we will detail it).

Quasi-doubly periodic case. The entries of elliptic $r$-matrix $\mathfrak{r}_{\lambda}^{+}(u)$ - elliptic Green distributions - are regularizations of quasi-doubly periodic functions. This is a direct consequence of those fact that this $r$-matrix satisfies Dynamical CYBE and therefore does not belong to the Belavin-Drinfeld classification [1]. Nevertheless, these functions have the elliptic type of the pole lattice $\mathfrak{R}=\Gamma=\mathbb{Z}+\mathbb{Z} \tau$ and one can expect that the $r$-matrix $\mathfrak{r}_{\lambda}^{+}(u)$ can be represented by formula (3.2) with $\gamma_{0}=\tau$ and $X(u)$ replaced by some trigonometric $r$-matrix with $\mathfrak{R}=\mathbb{Z}$. To pass on from the averaging of meromorphic functions to the averaging of distributions we should 
choose the proper regularization. Actually the regularization of this trigonometric $r$-matrix in this formula can depend on $n$ (see (3.7)). The $r$-matrices $r^{(a)+}(u, v), r^{(b)+}(u, v), r_{\lambda}^{+}(u, v)$ can be also regarded as a regularization of the same meromorphic $\mathfrak{s l}_{2} \otimes \mathfrak{s l}_{2}$-valued function, but they depend on $u, v$ in more general way than on the difference $(u-v)$. This makes the formalism of their averaging to be more complicated. Because of this reason we shall not consider these matrices in this paper.

Dynamical elliptic $r$-matrix as an averaging of $\mathfrak{r}^{(b) \pm}(u)$. To represent the $r$-matrix $\mathfrak{r}_{\lambda}^{+}(u)$ as an averaging of trigonometric matrix (2.7) we need the following formulae

$$
\begin{aligned}
\frac{\theta^{\prime}(u)}{\theta(u)} & =v \cdot p \cdot \sum_{n \in \mathbb{Z}} \pi \operatorname{ctg} \pi(u-n \tau) \\
\frac{\theta(u+\lambda)}{\theta(u) \theta(\lambda)} & =\frac{\theta^{\prime}(\lambda)}{\theta(\lambda)}+v \cdot p \cdot \sum_{n \in \mathbb{Z}}\left(\pi e^{-2 n \pi i \lambda} \operatorname{ctg} \pi(u-n \tau)+\left(1-\delta_{n 0}\right) \pi e^{-2 n \pi i \lambda} \operatorname{ctg} \pi n \tau\right)
\end{aligned}
$$

where $|\operatorname{Im} \lambda|<\operatorname{Im} \tau, \lambda \notin \mathbb{Z}$ and the symbol v.p. means convergence of the series in the principal value sense:

$$
\text { v.p. } \sum_{n \in \mathbb{Z}} x_{n}=\lim _{N \rightarrow \infty} \sum_{n=-N}^{N} x_{n}
$$

The Fourier expansion of the function $\frac{\theta^{\prime}(\lambda)}{\theta(\lambda)}$ has the form (1.8) (with $(u-z)$ replaced by $\lambda$ ) in the domain $-\operatorname{Im} \tau<\operatorname{Im} \lambda<0$. Substituting this expansion to the right hand side of (3.4) yields

$$
\begin{aligned}
& \frac{\theta(u+\lambda)}{\theta(u) \theta(\lambda)}=v \cdot p \cdot \sum_{n \in \mathbb{Z}} \pi e^{-2 n \pi i \lambda}(\operatorname{ctg} \pi(u-n \tau)+i) \\
& \frac{\theta(u-\lambda)}{\theta(u) \theta(-\lambda)}=v \cdot p \cdot \sum_{n \in \mathbb{Z}} \pi e^{2 n \pi i \lambda}(\operatorname{ctg} \pi(u-n \tau)-i)
\end{aligned}
$$

The formula (3.6) is obtained from (3.5) by replacing $u \rightarrow-u, n \rightarrow-n$, hence both formulae are valid in the domain $-\operatorname{Im} \tau<\operatorname{Im} \lambda<0$. Let us choose an automorphism $A=A_{\tau}$ as follows:

$$
A: h \mapsto h, \quad A: e \mapsto e^{2 \pi i \lambda} e, \quad A: f \mapsto e^{-2 \pi i \lambda} f
$$

and define $\vartheta_{n}=+$ for $n \geq 0$ and $\vartheta_{n}=-$ for $n<0$. Then the formulae (3.3), (3.5), (3.6) imply

$$
\mathfrak{r}_{\lambda}^{+}(u)=v \cdot p \cdot \sum_{n \in \mathbb{Z}}\left(A^{n} \otimes \mathrm{id}\right) \mathfrak{r}^{(b), \vartheta_{n}}(u-\tau n)
$$

where $-\operatorname{Im} \tau<\operatorname{Im} \lambda<0$ and $\mathfrak{r}^{(b),-}(u)$ are defined by formula $(2.7)$ with $\tilde{\psi}^{+}(u)$ replaced by $\tilde{\psi}^{-}(u)$. Let us notice that $r$-matrices $\mathfrak{r}_{\lambda}^{+}(u)$ and $\mathfrak{r}^{(b) \pm}(u)$ act as distributions on the same space $K$ and hence belong to the same space. Thus we do not have any problem with interpretation of the averaging formula in sense of distributions.

The matrix $\mathfrak{r}^{(c)+}(u)$ as an averaging of $\mathfrak{r}^{(a) \pm}(u)$. We restrict our attention to the case

$$
\frac{\operatorname{Im} \eta \operatorname{Im} \mu}{\operatorname{Re} \eta} \leq \operatorname{Re} \mu<\frac{\operatorname{Im} \eta \operatorname{Im} \mu}{\operatorname{Re} \eta}+1
$$

In this case Green distributions $\Psi_{\mu}^{+}(u), \Psi_{\mu}^{-}(-u), \Psi(u)$ entering into the $r$-matrix $\mathfrak{r}^{(c)+}(u)$ are defined by (2.9), (2.13). One has the formula

$$
2 \pi \eta \frac{e^{2 \pi \eta \mu u}}{e^{2 \pi \eta u}-1}=v \cdot p \cdot \sum_{n \in \mathbb{Z}} \frac{e^{2 \pi i \mu n}}{u-i \eta^{-1} n}
$$


Replacing $u \rightarrow-u, n \rightarrow-n$ in both sides yields

$$
2 \pi \eta \frac{e^{-2 \pi \eta \mu u}}{1-e^{-2 \pi \eta u}}=v \cdot p \cdot \sum_{n \in \mathbb{Z}} \frac{e^{-2 \pi i \mu n}}{u-i \eta^{-1} n}
$$

Let us choose the automorphism $A=A_{i \eta^{-1}}$ in the form

$$
A: H \mapsto H, \quad A: E \mapsto e^{2 \pi i \mu} E, \quad A: F \mapsto e^{-2 \pi i \mu} F
$$

Then the formulae imply the averaging $r$-matrix

$$
\mathfrak{r}^{(c)+}(u)=\sum_{n \in \mathbb{Z}}\left(A^{n} \otimes \mathrm{id}\right) \mathfrak{r}^{(a), \vartheta_{n}}\left(u-i \eta^{-1} n\right)
$$

where

$$
\frac{\operatorname{Im} \eta \operatorname{Im} \mu}{\operatorname{Re} \eta} \leq \operatorname{Re} \mu<\frac{\operatorname{Im} \eta \operatorname{Im} \mu}{\operatorname{Re} \eta}+1
$$

and $\mathfrak{r}^{(a),-}(u)$ is defined by formula $(2.5)$ with $\Phi^{+}(u)$ substituted by $\Phi^{-}(u)$. The $r$-matrices $\mathfrak{r}^{(c)+}(u)$ and $\mathfrak{r}^{(a) \pm}(u)$ act also on the same space - on the algebra $Z$ from subsection 2.2.

\section{Appendix: Test function algebras $\mathcal{K}_{0}$ and $K=K(\mathrm{Cyl})$}

Test function algebra $\mathcal{K}_{0}$. Let $\mathcal{K}_{0}$ be a set of complex-valued meromorphic functions defined in some vicinity of origin which have an only pole in the origin. If $s_{1}(u)$ and $s_{2}(u)$ are two such functions with domains $U_{1}$ and $U_{2}$ then their sum $s_{1}(u)+s_{2}(u)$ and their product $s_{1}(u) \times s_{2}(u)$ are also functions of this type which are defined in the intersection $U_{1} \cap U_{2}$. Moreover if $s(u)$ is a function from $\mathcal{K}_{0}$ which is not identically zero then there exists a neighborhood $U$ of the origin such that the domain $U \backslash\{0\}$ does not contain zeros of function $s(u)$ and, therefore, the function $\frac{1}{s(u)}$ is a function from $\mathcal{K}_{0}$ with the domain $U$. This means that the set $\mathcal{K}_{0}$ can be endowed with a structure of a field. We shall consider $\mathcal{K}_{0}$ as an associative unital algebra over $\mathbb{C}$ equipped with the invariant scalar product

$$
\left\langle s_{1}(u), s_{2}(u)\right\rangle=\oint_{C_{0}} \frac{d u}{2 \pi i} s_{1}(u) s_{2}(u)
$$

where $C_{0}$ is a contour encircling zero and belonging to the intersection of domains of functions $s_{1}(u), s_{2}(u)$, such that the scalar product is a residue in zero. We consider the algebra $\mathcal{K}_{0}$ as an algebra of test functions. A convergence in $\mathcal{K}_{0}$ is defined as follows: a sequence of functions $\left\{s_{n}(u)\right\}$ converges to zero if there exists a number $N$ such that all the function $z^{N} s_{n}(u)$ are regular in origin and all the coefficients in their Laurent expansion tend to zero. One can consider, instead of the algebra $\mathcal{K}_{0}$ defined in this way, the completion $\overline{\mathcal{K}}_{0}=\mathbb{C}\left[u^{-1}\right][[u]]$. Linear continuous functionals on $\mathcal{K}_{0}$ are called distributions and form the space $\mathcal{K}_{0}^{\prime}$ which coincide with $\overline{\mathcal{K}}_{0}^{\prime}$. The scalar product (4.1) being continuous defines a continuous injection $\mathcal{K}_{0} \rightarrow \mathcal{K}_{0}^{\prime}$. We use the notation $\langle a(u), s(u)\rangle$ for the action of a distribution $a(u)$ on a test function $s(u)$ and also the notation $\langle a(u)\rangle_{u}=\langle a(u), 1\rangle$, where 1 is a function which identically equals to the unit.

One can define a 'rescaling' of a test function $s(u)$ as a function $s\left(\frac{u}{\alpha}\right)$, where $\alpha \in \mathbb{C}$, and therefore a 'rescaling' of distributions by the formula $\left\langle a\left(\frac{u}{\alpha}\right), s(u)\right\rangle=\langle a(u), s(\alpha u)\rangle$. On the contrary, we are unable to define a 'shift' of test functions by a standard rule, because the 
operator $s(u) \mapsto s(u+z)$ is not a continuous one ${ }^{2}$. Nevertheless we use distributions 'shifted' in some sense. Namely, we say that a two-variable distribution $a(u, z)$ (a linear continuous functional $a: \mathcal{K}_{0} \otimes \mathcal{K}_{0} \rightarrow \mathbb{C}$ ) is 'shifted' if it possesses the properties: (i) for any $s \in \mathcal{K}_{0}$ the functions $s_{1}(z)=\langle a(u, z), s(u)\rangle_{u}$ and $s_{2}(u)=\langle a(u, z), s(z)\rangle_{z}$ belong to $\mathcal{K}_{0}$; (ii) $\frac{\partial}{\partial u} a(u, z)=$ $-\frac{\partial}{\partial z} a(u, z)$. Here the subscripts $u$ and $z$ mean the corresponding partial action, for instance, $\langle a(u, z), s(u, z)\rangle_{u}$ is a distribution acting on $\mathcal{K}_{0}$ by the formula

$$
\left\langle\langle a(u, z), s(u, z)\rangle_{u}, t(z)\right\rangle=\langle a(u, z), s(u, z) t(z)\rangle
$$

The condition (ii) means the equality $\left\langle a(u, z), s^{\prime}(u) t(z)\right\rangle=-\left\langle a(u, z), s(u) t^{\prime}(z)\right\rangle$. The condition (i) implies that for any $s \in \mathcal{K}_{0} \otimes \mathcal{K}_{0}$ the expression

$$
\langle a(u, z), s(u, z)\rangle_{u}=\sum_{i}\left\langle a(u, z), p_{i}(u)\right\rangle_{u} q_{i}(z)
$$

where $s(u, z)=\sum_{i} p_{i}(u) q_{i}(z)$, belongs to $\mathcal{K}_{0}$ (as a function of $z$ ).

Semidirect product. Now we are able to define a semidirect product of two 'shifted' distributions $a(u, z)$ and $b(v, z)$ as a linear continuous functional $a(u, z) b(v, z)$ acting on $s \in \mathcal{K}_{0} \otimes \mathcal{K}_{0} \otimes \mathcal{K}_{0}$ by the rule

$$
\langle a(u, z) b(v, z), s(u, v, z)\rangle=\left\langle a(u, z),\langle b(v, z), s(u, v, z)\rangle_{v}\right\rangle_{u, z}
$$

The 'shifted' distribution $a(u, z)$ acting on $\mathcal{K}_{0} \otimes \mathcal{K}_{0}$ can be defined by one of its partial actions on the function of one variable. For instance, if the partial action of the type $\langle a(u, v), s(u)\rangle_{u}$ is defined for any test function $s(u)$ then one can calculate the left hand side of (4.2) and, then, obtain the total action of $a(u, z)$ on the test function $s(u, z)$. This means that a 'shifted' distribution (more generally, a distribution satisfying condition (i)) define a continuous operator on $\mathcal{K}_{0}$.

The main example of a 'shifted' distribution is a delta-function $\delta(u, z)$ defined by one of the formulae

$$
\langle\delta(u, z), s(u)\rangle_{u}=s(z), \quad\langle\delta(u, z), s(z)\rangle_{z}=s(u), \quad\langle\delta(u, z), s(u, z)\rangle_{u, z}=\langle s(z, z)\rangle_{z}
$$

It is symmetric, $\delta(u, z)=\delta(z, u)$, and one can show that any 'shifted' distribution $a(u, v)$ satisfies

$$
a(u, v) \delta(u, z)=a(z, v) \delta(u, z)
$$

The distribution $\delta(u, z)$ defines an identical operator on $\mathcal{K}_{0}$.

Test function algebra $K$. We define the algebra $K$ as an algebra of entire functions on $\mathbb{C}$ subjected to the periodicity condition $s(u+1)=s(u)$ and to the condition $|s(u)| \leq C e^{p|\operatorname{Im} u|}$, where the constants $C, p>0$ depend on the function $s(u)$. The periodicity of these functions means that they can be considered as functions on the cylinder $\mathrm{Cyl}=\mathbb{C} / \mathbb{Z}: K=K(\mathrm{Cyl})$. We equip the algebra $K$ with an invariant scalar product

$$
\langle s(u), t(u)\rangle=\int_{-\frac{1}{2}+\alpha}^{\frac{1}{2}+\alpha} \frac{d u}{2 \pi i} s(u) t(u), \quad s, t \in K
$$

which does not depend on a choice of the complex number $\alpha \in \mathbb{C}$. A convergence in $K$ is given as follows: a sequence $\left\{s_{n}\right\} \subset K$ tends to zero if there exist such constants $C, p>0$ that

\footnotetext{
${ }^{2}$ Consider, for example, the sum $s_{N}(u)=\sum_{n=0}^{N}\left(\frac{u}{\alpha}\right)^{n}$. For each $z$ there exist $\alpha$ such that the sum $s_{N}(u+z)$ diverges, when $N \rightarrow \infty$.
} 
$\left|s_{n}(u)\right| \leq C e^{p|\operatorname{Im} u|}$ and $s_{n}(u) \rightarrow 0$ for all $u \in \mathbb{C}$. In particular, if $s_{n} \rightarrow 0$ then the functions $s_{n}(u)$ tends uniformly to zero. Therefore, the scalar product (4.4) is continuous with respect to this topology and it defines a continuous embedding of $K=K(\mathrm{Cyl})$ to the space of distributions $K^{\prime}=K^{\prime}(\mathrm{Cyl})$.

Each function $s \in K$ can be restricted to the line segment $\left[-\frac{1}{2}+\alpha ; \frac{1}{2}+\alpha\right]$, be expanded in this line segment to a Fourier series and, then, this expansion can be uniquely extended to all the $\mathbb{C}$ by the analyticity principle. It means that $\left\{j_{n}(u)=e^{2 \pi i n u}\right\}_{n \in \mathbb{Z}}$ is a basis of $K$ and $\left\{j^{n}(u)=2 \pi i e^{-2 \pi i n u}\right\}_{n \in \mathbb{Z}}$ is its dual one with respect to the scalar product (4.4).

The functions belonging to the space $K$ can be correctly shifted because for all $z \in \mathbb{C}$ the operator $\mathcal{T}_{z}: s(u) \mapsto s(u+z)$ is continuous and maps a periodic function to a periodic one. Hence we can define sifted distributions in the usual way: $\langle a(u-z), s(u)\rangle=\langle a(u), s(u+z)\rangle$. Thereby defined shifted distributions $a(u-z)$ can be considered as two-variable distributions with properties (i) and (ii) as well as distributions depending on one of variables as an argument and on another as a parameter. For example the distribution $\delta(u) \in K^{\prime}$ defined by the formula $\langle\delta(u), s(u)\rangle=s(0)$ can be shifted by variable $z$ and considered as a distribution of variables $u$ and $z$. This shifted distribution is called delta-function. Its Fourier expansion looks as follows

$$
\delta(u-z)=\sum_{n \in \mathbb{Z}} j^{n}(u) j_{n}(z)=2 \pi i \sum_{n \in \mathbb{Z}} e^{-2 \pi i n(u-z)}
$$

\section{Acknowledgements}

This paper is a part of $\mathrm{PhD}$ thesis of $\mathrm{A}$. S. which he is prepared under co-supervision of S. P. and V. R. in the Bogoliubov Laboratory of Theoretical Physics, JINR, Dubna and in LAREMA, Département de Mathématics, Université d'Angers. He is grateful to the CNRS-Russia exchange program on mathematical physics and personally to J.-M. Maillet for financial and general support of this thesis project. V.R. is thankful to B.Enriquez for discussions. He had used during the project a partial financial support by ANR GIMP, Grant for support of scientific schools NSh-8065.2006.2 and a support of INFN-RFBR "Einstein" grant (Italy-Russia). He acknowledges a warm hospitality of Erwin Schrödinger Institute for Mathematical Physics and the Program "Poisson Sigma Models, Lie Algebroids, deformations and higher analogues" where this paper was finished. S.P. was supported in part by RFBR grant 06-02-17383.

\section{References}

[1] A. A. Belavin and V. G. Drinfeld. Solutions of the classical Yang-Baxter equation for simple Lie algebras. Funct. Anal. Appl., 16 (1983), 159-180.

[2] B. Enriquez and G. Felder. Elliptic quantum groups $E_{\tau, \eta}\left(\mathfrak{s l}_{2}\right)$ and quasi-Hopf algebras. Comm. Math. Phys., 195 (1998), 651-689.

[3] B. Enriquez and V. Rubtsov. Quantum groups in higher genus and Drinfeld's new realizations method ( $\mathfrak{s l}_{2}$ case). Ann. Sci. École Norm. Sup. (4) 30 (1997), 821-846.

[4] L. D. Faddeev and N. Yu. Reshetikhin. Hamiltonian structures for integrable models of field theory. Theoret. and Math. Phys., 56 (1983), 847-862.

[5] G. Felder. Conformal field theory and integrable systems associated to elliptic curves. Proc. ICM Zürich 1994, 1247-1255, Birkhäuser (1994).

[6] G. Felder. Elliptic quantum groups. Proc. ICMP Paris 1994, 211-218, International Press (1995).

[7] I. M. Gelfand and E. G. Shilov. Generalized Functions. Vol. I. Academic Press, New York, 1964.

[8] S. Khoroshkin. Central extension for the Yangian double. Algèbre non commutative, groupes quantiques et invariants. Reims, 1995, 119-135, Sémin. Congr. 2, Soc. Math. France, Paris, 1997.

[9] S. Khoroshkin, D. Lebedev, and S. Pakuliak. Elliptic algebra in the scaling limit. Comm. Math. Phys. 190 (1998), 597-627. 
[10] S. Khoroshkin, D. Lebedev, and S. Pakuliak. Yangian algebras and classical Riemann problem. In "Moscow Seminar in Mathematical Physics", 163-198; Amer. Math. Soc. Transl. Ser. 2, 191, Amer. Math. Soc., Providence, RI, 1999.

[11] S. Khoroshkin, D. Lebedev, S. Pakuliak, A. Stolin, and V. Tolstoy. Classical limit of the scaled elliptic algebra. Compos. Math. 115 (1999), 205-230.

[12] H. Konno. An elliptic algebra $U_{q, p}\left(\widehat{\mathfrak{s l}}_{2}\right)$ and the fusion RSOS model. Comm. Math. Phys. 195 (1998), 373-403.

[13] A. LeClair, S. Khoroshkin, and S. Pakuliak. Angular quantization of the Sine-Gordon model at the free fermion point. Adv. Theor. Math. Phys. 3 (1999), 1227-1287.

[14] S. Pakuliak, V. Rubtsov, and A. Silantyev. Classical elliptic current algebras. I. J. Gen. Lie Theory Appl. 2 (2008), 65-78.

[15] V. S. Vladimirov. Methods of the Theory of Generalized Functions. Taylor and Francis Ltd., 2002.

Received September 28, 2007

Revised December 09, 2007 